\title{
Evaluación del tratamiento preliminar y primario para las aguas residuales del procesamiento industrial de alimentos en La Grita (Venezuela)
}

\author{
Evaluation of the preliminary and primary \\ treatment for wastewater from industrial food \\ processing in La Grita (Venezuela)
}

DOI: http://doi.org/10.17981/ingecuc.17.1.2021.01

Artículo de Investigación Científica. Fecha de Recepción: 06/07/2020. Fecha de Aceptación: 16/10/2020.

\author{
Kleiver Pimiento (1) \\ Alfatlantic C.A. La Grita (Venezuela) \\ kleiver.pimiento.28@gmail.com \\ Marco José Cárdenas González (1) \\ Universidad Nacional Experimental del Táchira. San Cristóbal (Venezuela) \\ mjcardenas14@gmail.com
}

Para citar este artículo:

K. Pimiento \& M. Cárdenas González, "Evaluación del tratamiento preliminar y primario para las aguas residuales del procesamiento industrial de alimentos en La Grita (Venezuela)", INGE CUC, vol. 17, no. 1, pp. 1-14. DOI: http://doi. org/10.17981/ingecuc.17.1.2021.01

\section{Resumen}

Introducción - La crisis venezolana ha disminuido la producción industrial, con la consecuente merma en el caudal y cambios en la calidad de las aguas residuales que se generan, afectando los sistemas de depuración de efluentes.

Objetivo- Evaluar los tratamientos preliminares y primarios del agua residual en una industria de alimentos ubicada en Táchira (Venezuela) y presentar alternativas de mejora.

Metodología- Se evaluaron el tanque de bombeo, homogeneizador y Flotación por Aire Disuelto (DAF) Para ello se tomaron muestras de agua y se determinaron parámetros de calidad, los cuales se compararon con lo previsto en el diseño. Se realizaron cálculos para verificar parámetros técnicos del diseño y de la literatura.

Resultados - El caudal tratado oscila entre 0.95 y $2.84 \mathrm{~L} / \mathrm{s}$, versus los $12.16 \mathrm{~L} / \mathrm{s}$ del diseño. Esto ha aumentado el tiempo de retención en las unidades de tratamiento, lo cual, a su vez, disminuye el $\mathrm{pH}$ del agua desde $6.10 \pm 0.23$ hasta $5.34 \pm 0.23$ por la ocurrencia de procesos anaeróbicos, afectándose la coagulación. El DAF es eficiente y disminuye $79 \%$ los SST y un $62 \%$ la DBO5.20, pero remueve el N y P hasta $2.5 \pm 1 \mathrm{mg} / \mathrm{L}$ y $9 \pm 4 \mathrm{mg} / \mathrm{L}$, respectivamente, resultando insuficiente la relación de nutrientes necesaria en el tratamiento biológico.

Conclusiones- El diseño del sistema de tratamiento tuvo como error la calidad teórica del agua que se asumió. La merma de caudal afecta el tratamiento preliminar y primario. Se deben tomar medidas de mejora en la coagulación y floculación del DAF.

Palabras clave - Agua residual industrial; coagulación; evaluación; flotación por aire disuelto; homogeneizador; planta de tratamiento; tratamiento primario

\begin{abstract}
Introduction-The Venezuelan crisis has decreased industrial production, with the consequent decrease in flow and changes in the quality of the wastewater generated, affecting the effluent treatment systems.

Objective - Evaluate the preliminary and primary treatments of residual water in a food industry located in Táchira (Venezuela) and present alternatives for improvement.
\end{abstract}

Methodology - The pumping tank, homogenizer and Dissolved Air Flotation (DAF) were evaluated. For this, water samples were taken and quality parameters were determined, which were compared with what was foreseen in the design. Calculations were performed to verify technical parameters contemplated in the design and literature.

Results - The flow treated ranges from 0.95 to 2.84 $\mathrm{L} / \mathrm{s}$, versus the $12.16 \mathrm{~L} / \mathrm{s}$ of the design. This has increased the retention time in the treatment units, which, in turn, decreases the $\mathrm{pH}$ of the water from $6.10 \pm 0.23$ to $5.34 \pm 0.23$ due to the occurrence of anaerobic processes, affecting the coagulation. The DAF is efficient and decreases $79 \%$ the TSS and $62 \%$ the BOD5.20, but removes the $\mathrm{N}$ and $\mathrm{P}$ up to $2.5 \pm 1 \mathrm{mg} / \mathrm{L}$ and $9 \pm 4 \mathrm{mg} / \mathrm{L}$, respectively, resulting in insufficient nutrients ratio necessary in biological treatment. Conclusions: The design of the treatment system had as an error the theoretical quality of the water that was assumed. The decrease in flow affects the preliminary and primary treatment. Improvement measures must be taken in the coagulation and flocculation of the DAF.

Keywords- Industrial waste water; coagulation; evaluation; dissolved air flotation; homogenizer; waste water treatment plant; primary treatment 


\section{INTRODUCCIÓN}

Las plantas de tratamiento de agua residual se seleccionan y diseñan para caudales y calidad física, química y microbiológica que se encuentren en rangos específicos; es decir, para una determinada capacidad [1]. No obstante, en la realidad operativa de estos sistemas se puede denotar: elementos físicos ausentes, inconclusos o deteriorados; contingencias no previstas o que superan lo esperado; cambios en los procesos generadores de los efluentes o aumento de la demanda de tratamiento; debilidades en la operación y mantenimiento; entre otros [2], [3], [4], [5], [6]. Todo lo anterior, junto a errores u omisiones en el diseño [6], [7] y ausencia de estudios de calidad de los vertidos y de potenciales tecnologías en plantas pilotos, puede ocasionar que el funcionamiento de los sistemas de depuración existentes se aleje de lo previsto en los proyectos originales.

En la ciudad de La Grita, municipio Jáuregui del estado Táchira (Venezuela), existe una industria encargada de la producción de botanas o alimentos tipo snack, cuyas actividades han disminuido en los últimos años por la crítica situación socioeconómica del país. Esto ha generado, entre otras cosas, menor cantidad de agua residual y cambios en su composición [8], alejándose de las especificaciones tomadas para elaborar el diseño original de la Planta de Tratamiento de Agua Residual (PTAR) industrial existente. Esta variación ha ocasionado que se evidencien afectaciones negativas dentro del sistema de depuración, como (a) la generación de malos olores en las unidades de tratamiento preliminar y primario de la PTAR, (b) visualización de sustancias químicas remanentes de la coagulación y floculación en el efluente del proceso de Flotación por Aire Disuelto (DAF) y, sobre todo, (c) incumplimiento de la norma de descargas nacional y de las estipulaciones internas de la multinacional.

Evaluar el sistema de tratamiento llevando la secuencia de las distintas operaciones y procesos existentes, fue una necesidad lógica que permitiera tomar acciones de mejoras y lograr el cumplimiento de los límites de descargas. En esta publicación se muestran los resultados de dicha evaluación y las alternativas para el mejoramiento de las primeras unidades de depuración existentes en el sistema objeto de estudio: tanque de bombeo, tanque de igualación u homogeneización, y DAF con inyección de químicos, entendiendo que mejoras en las tecnologías antecesoras van a afectar positivamente en los procesos subsiguientes y en la calidad del vertido final.

\section{Metodología}

Las aguas residuales son generadas en procesos de elaboración de alimentos tipo snacks o botanas. Al momento de la investigación, las líneas que se mantenían produciendo eran: aros de maíz dulces, palitos de maíz horneados (dos productos diferentes: blandos y duros) y galletas de trigo horneadas. Los efluentes residuales que se generan en la producción industrial son conducidos hacia una PTAR que consta de operaciones físicas y procesos químicos y biológicos (Fig. 1). La presente evaluación se desarrolló por fases, siguiendo la secuencia lógica del tren de tratamiento. En este escrito se presenta sólo lo concerniente a las primeras unidades de tratamiento: tanque de bombeo, tanque de igualación, cuba de coagulación, floculador mecánico y flotación por aire disuelto. Los tratamientos preliminares y primarios fueron la unidad de estudio o caso de análisis.

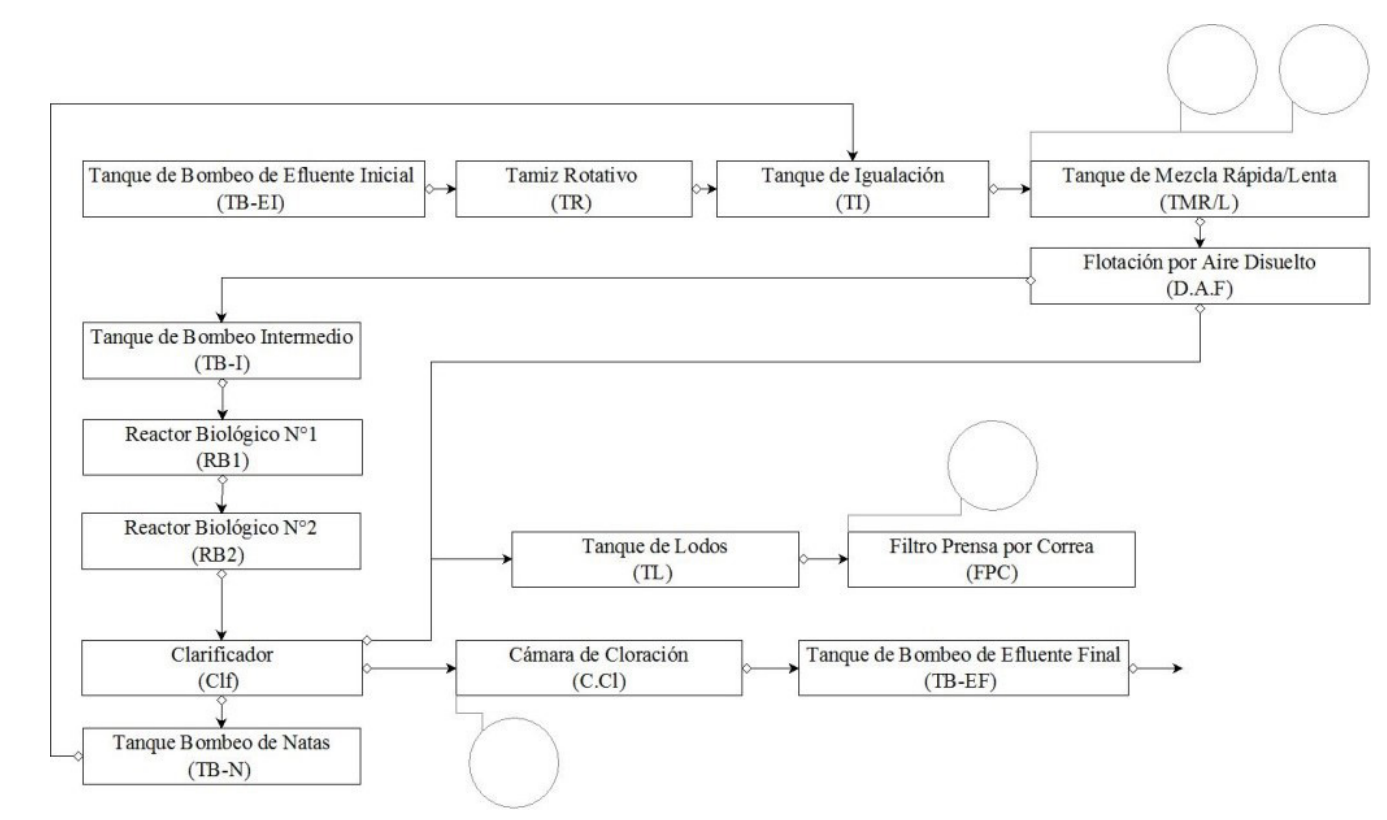

Fig. 1. Diagrama de la planta de tratamiento de agua residual industrial objeto de estudio. Fuente: [8]. 
Desde julio del 2018 hasta marzo de 2019, se efectuó la medición de caudal y toma de muestras del agua residual en diversos puntos de interés: afluente y efluente del tanque de bombeo, efluentes del tanque de igualación y DAF. El caudal se cuantificó gracias a un caudalímetro existente en la conducción afluente al DAF.

En el caso de los análisis físicos y químicos hechos al agua residual, se siguieron los procedimientos, reactivos (kits) y equipos HANNA Instruments ${ }^{\circledR}$ : $\mathrm{pH}$ metro HI8424 (con la finalidad de conseguir el pH), fotómetro multiparamétrico C99 y reactor HI839800 (demanda química de oxígeno o DQO); junto con el colorímetro $\mathrm{HACH}^{\circledR} \mathrm{DR} / 890$ para las pruebas de sólidos suspendidos totales (SST), nitrógeno y fósforo, y el uso del OxiTop ${ }^{\circledR}$ Box de la marca WTW para hallar la $\mathrm{DBO}_{5.20}$ (demanda bioquímica de oxígeno a los cinco días y $20^{\circ} \mathrm{C}$ ) (Fig. 2).

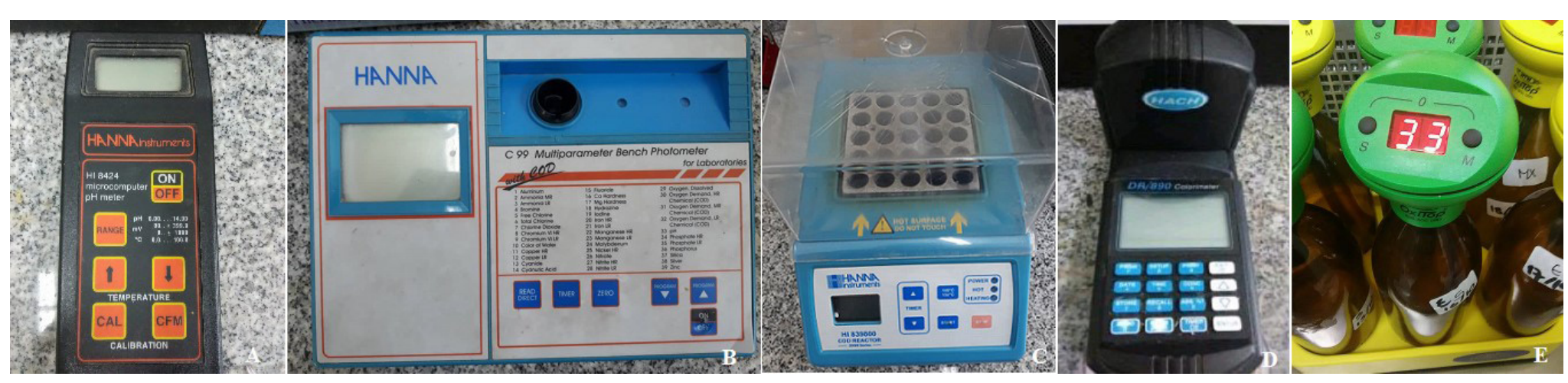

Fig. 2. Equipos utilizados para medir pH (A), DQO (B y C), sólidos suspendidos totales, nitrógeno y fósforo (D), DBO5,20 (E). Fuente: Autores.

Dichos parámetros y equipos utilizados se fundamentan en los métodos: electrométrico o potenciométrico $(\mathrm{pH})$, reflujo cerrado colorimétrico (DQO), colorimetría (SST, nitrógeno y fósforo) y respirométrico o manométrico $\left(\mathrm{DBO}_{5.20}\right)$ [9], [10], [11].

Los parámetros se determinaban cada hora a muestras simples de agua residual en los mencionados puntos. Adicionalmente, con 24 alícuotas de las tomas efectuadas cada hora, se preparaban y analizaban tres réplicas de una muestra compuesta diaria y, finalmente, se calculaba el promedio. En la investigación se reporta la media de todas las muestras compuestas. Todos los parámetros fueron determinados en el Laboratorio de la PTAR de la empresa (laboratorio interno).

Se realizaron algunas pruebas de tratabilidad para evaluar el funcionamiento de los procesos de coagulación y floculación y brindar opciones de mejoras. Estos ensayos (pruebas de jarras) se adaptaron de [12], para lo cual se aplicaron $3 \mathrm{~mL}$ de sulfato de aluminio $\left(\mathrm{Al}_{2}\left(\mathrm{SO}_{4}\right)_{3}\right.$ al $\left.4 \%\right)$ a $500 \mathrm{~mL}$ de agua residual, se agitaron a $150 \mathrm{RPM}$ en un equipo para pruebas de jarras durante 1 min, luego 5 min a 50 RPM y, finalmente, se dejó reposar a lo largo de 10 min. También se hicieron pruebas variando los tiempos y el mezclado para visualizar la formación de flóculos y su rompimiento.

También se efectuaron cálculos con la finalidad de verificar el cumplimiento de parámetros de diseño en las unidades de depuración. Se destacan la determinación del tiempo de retención hidráulico (1), relaciones de nutrientes (2, 3, 4 y 5), carga hidráulica superficial (6), cuyas ecuaciones se adaptaron [13], [14].

$$
\begin{aligned}
& \theta=\frac{V}{Q} \\
& D B O_{5,20} / N / P=100 / A / B \\
& C=\frac{D B O_{5,20}}{100} \\
& A=\frac{N}{C}
\end{aligned}
$$




$$
\begin{aligned}
& B=\frac{P}{C} \\
& C H S=\frac{Q}{A_{S}}
\end{aligned}
$$

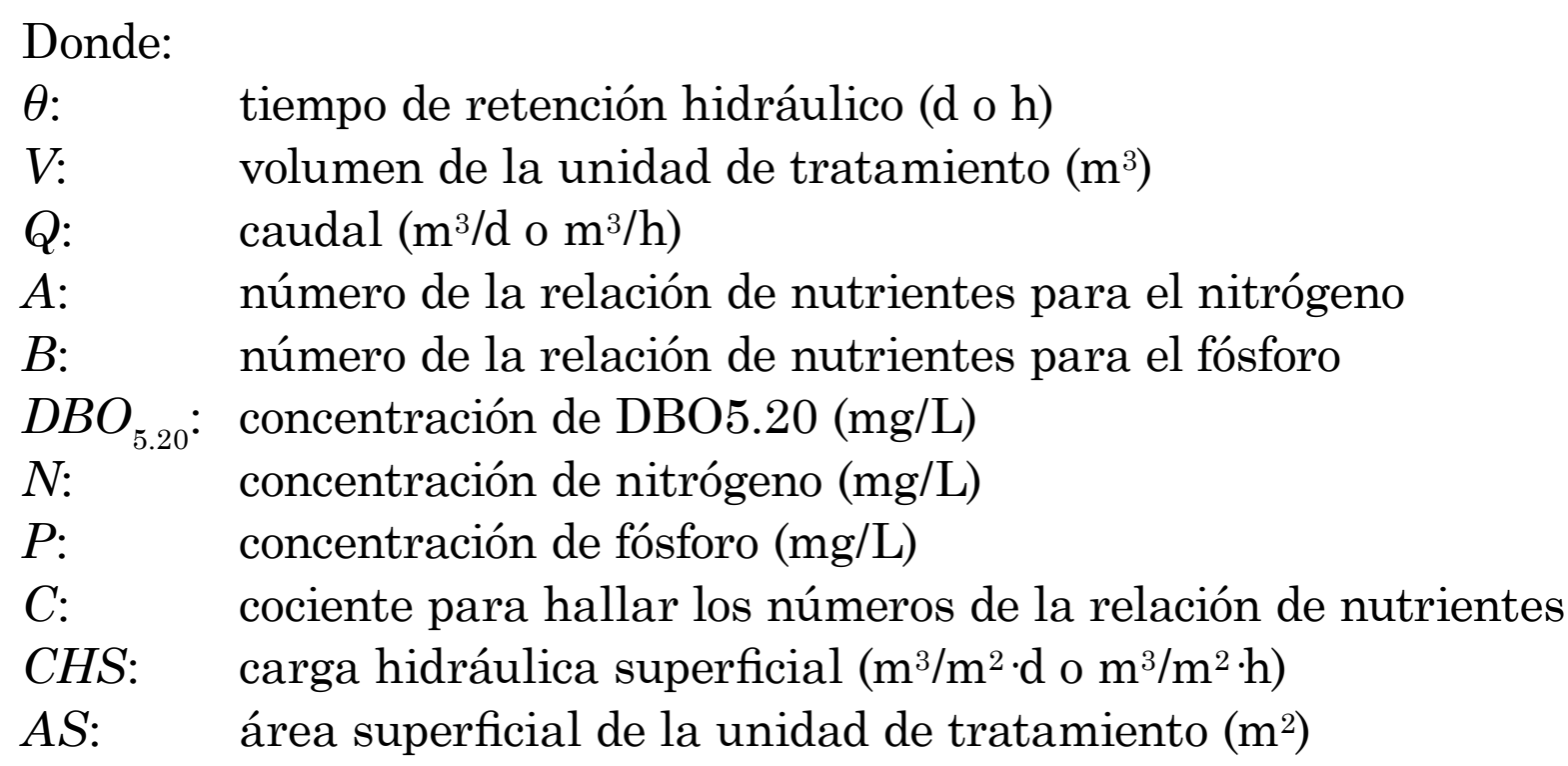

\section{REsultados y Discusión}

\section{A. Parámetros originales de diseño}

El sistema de tratamiento se diseñó para depurar un caudal promedio de $12.16 \mathrm{~L} / \mathrm{s}$ de agua residual industrial [15] proveniente de distintos procesos donde se elaboran snacks o botanas. Tanto el caudal como las concentraciones de diseño (Tabla 1), fueron proporcionados por la multinacional propiedad de la planta de producción en La Grita (Venezuela).

Tabla 1. Parámetros para el diseño original de la PTAR.

\begin{tabular}{|l|l|l|}
\hline \multicolumn{1}{|c|}{ Parámetros } & \multicolumn{1}{c|}{ Unidades } & \multicolumn{1}{c|}{ Valor de diseño } \\
\hline Caudal medio & $\mathrm{L} / \mathrm{s}$ & 12.16 \\
\hline $\mathrm{DBO}_{5.20}$ & $\mathrm{mg} / \mathrm{L}$ & 4334.00 \\
\hline DQO & $\mathrm{mg} / \mathrm{L}$ & 8365.00 \\
\hline DQO/DBO & - & 1.93 \\
\hline SST & $\mathrm{mg} / \mathrm{L}$ & 8657.00 \\
\hline Nitrógeno total & $\mathrm{mg} / \mathrm{L}$ & 62.00 \\
\hline Fósforo total & $\mathrm{mg} / \mathrm{L}$ & 3.00 \\
\hline Carga de DBO & $\mathrm{kg} / \mathrm{d}$ & 1794.00 \\
\hline Carga de DQO & $\mathrm{kg} / \mathrm{d}$ & 9095.00 \\
\hline Carga de SST & $\mathrm{kg} / \mathrm{d}$ & 3584.00 \\
\hline
\end{tabular}

Nota: DBO5.20: Demanda Bioquímica de Oxígeno medida a los cinco días y a $20^{\circ} \mathrm{C}$, DQO: Demanda Química de Oxígeno, SST: Sólidos Suspendidos Totales.

Fuente: [15].

Los resultados obtenidos en el proceso de evaluación se presentan por separado para cada unidad de tratamiento.

\section{B. Tanque de Bombeo y Unidad de Igualamiento}

Las aguas residuales industriales pasan, primeramente, por una reja metálica de desbaste de limpieza manual. De allí el líquido discurre a un tanque de bombeo (Fig. 3) cuya única función es trasvasarlo hasta el homogeneizador debido a la imposibilidad de que suceda por gravedad. El tanque de bombeo está conformado por dos estructuras adosadas, subterráneas y construidas en concreto. Cada uno cuenta con un sistema de dos bombas sumergibles; un sistema de control de bollas y panel de control centralizado, para manipulación en manual, off, automático; tienen un mecanismo de polipasto que permite la elevación de los equipos de bombeo; y una compuerta 
manual para el ingreso del agua. La capacidad efectiva de cada fosa es de $27 \mathrm{~m}^{3}$, lo cual se traduce en un tiempo de retención de $0.62 \mathrm{~h}$ para los $12.16 \mathrm{~L} / \mathrm{s}$ de agua residual previstos en el diseño original. Sin embargo, la realidad es que el caudal que ingresa al tanque de bombeo oscila entre 2.84 y $0.95 \mathrm{~L} / \mathrm{s}$ (entre un 7.8 y $23 \%$ de lo contemplado en el diseño), ocasionando tiempos de retención que van de $2.6 \mathrm{~h}$ a $8 \mathrm{~h}$, respectivamente. Esto significa que el agua dentro del tanque permanece mucho más tiempo del contemplado en el proyecto original (de 4.19 a 12.90 veces).
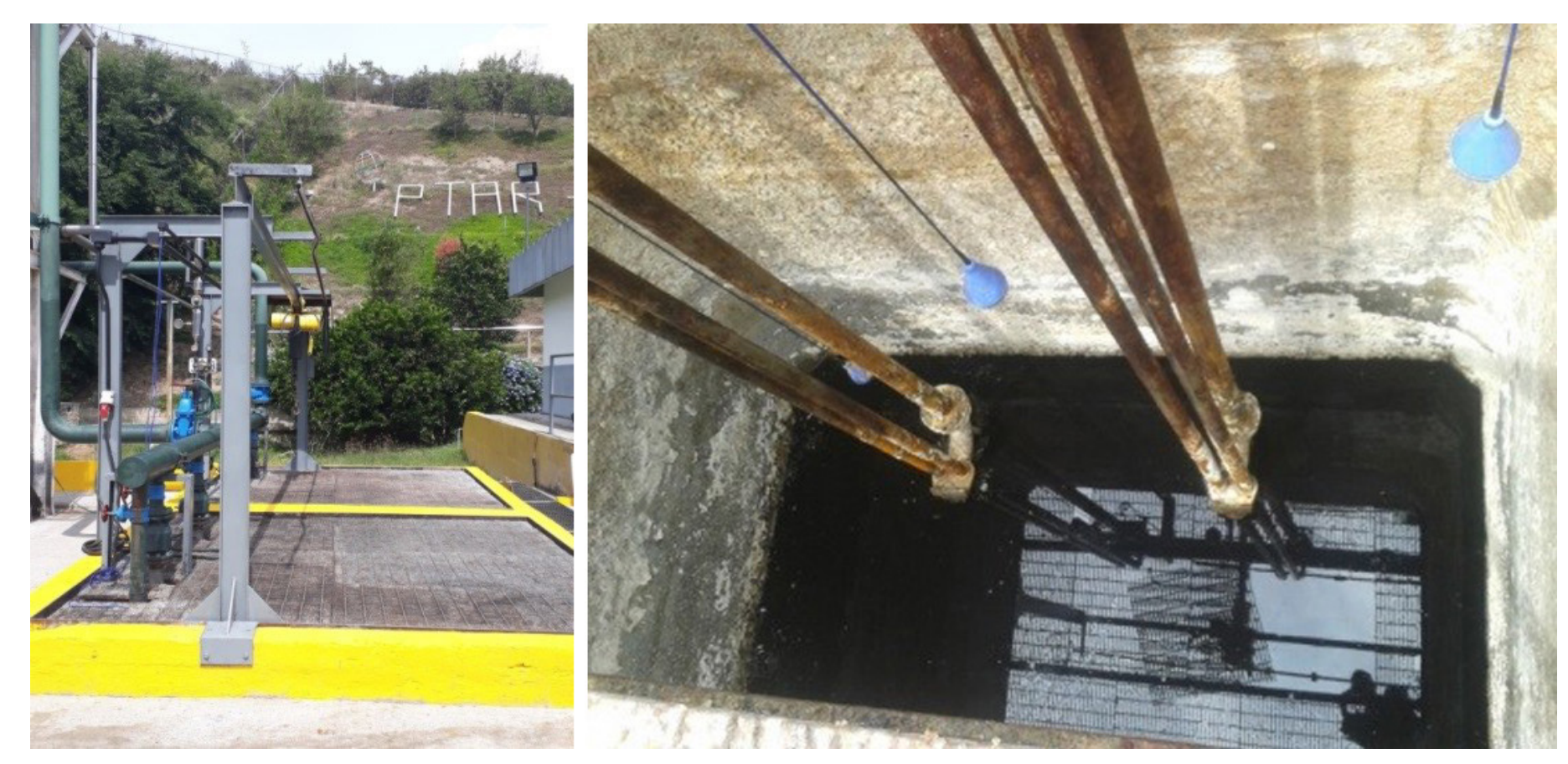

Fig. 3. Vista exterior e interior del tanque de bombeo. Fuente: Autores.

Desde el punto de vista de trasladar el agua de un punto a otro, lo anterior es irrelevante y no ocasiona afectaciones. No obstante, se destacan las implicaciones que el almacenamiento prolongado puede provocar en la calidad del líquido, por ejemplo en su pH (Tabla 2). La disminución de este parámetro y el elevado tiempo de retención existente, pueden asociarse a procesos de oxidación anaeróbica de la materia orgánica presente en el agua residual.

Tabla 2. Registro de PH del afluente en El tanque DE Bombeo y TANQUE DE IGUALACIÓN DESDE OCTUBRE 2017- MAYO 2018

\begin{tabular}{|l|l|l|l|}
\hline Línea de producción & \multicolumn{1}{|c|}{ Afluente } & Tanque de bombeo & \multicolumn{1}{c|}{ Tanque de igualación } \\
\hline Rosquis & $6.40 \pm 0.30$ & $6.02 \pm 0.08$ & $5.08 \pm 0.13$ \\
\hline Twistos & $6.03 \pm 0.12$ & $6.00 \pm 0.25$ & $5.68 \pm 0.10$ \\
\hline Pepito & $6.10 \pm 0.10$ & $6.00 \pm 0.03$ & $5.40 \pm 0.21$ \\
\hline Cheese tris & $6.00 \pm 0.07$ & $6.00 \pm 0.12$ & $5.32 \pm 0.08$ \\
\hline Cocimiento de maíz & $5.97 \pm 0.21$ & $5.95 \pm 0.07$ & $5.24 \pm 0.11$ \\
\hline Promedio & $6.10 \pm 0.23$ & $5.99 \pm 0.14$ & $5.34 \pm 0.23$ \\
\hline
\end{tabular}

Fuente: Autores.

De hecho, dentro de la descomposición anaeróbica, la primera fase que ocurre es la hidrólisis, en la cual las moléculas más grandes o polímeros (carbohidratos, proteínas, lípidos...) son convertidos en sus respectivos constitutuyentes individuales (monómeros): monosacáridos, aminoácidos y ácidos grasos. Como segunda fase, ocurre la acidogénesis, donde los monómeros se descomponen en ácidos orgánicos de cadena corta como el ácido butírico, propiónico, acético, hidrógeno y dióxido de carbono [16], [17], [18]. Debido a la formación de los mencionados ácidos, se ha demostrado que el pH del sustrato disminuirá [16], sobre todo cuando en la materia orgánica abundan los carbohidratos [18]. Siendo el maíz rico en carbohidratos [19] y una de las principales materias primas de la industria que genera las aguas residuales en estudio, no es de extrañar el comportamiento descrito. Asimismo, los marcados malos olores generados en las unidades de tratamiento, fueron otra evidencia de la ocurrencia de descomposición anaeróbica. 
La reducción del potencial de hidrógeno existente entre la descarga y el tanque de bombeo es mínima (coincidiendo con la etapa anaerobia mencionada), pero es mayor al compararse con los valores dentro del tanque de igualación. Éste permite la homogeneización de corrientes y el almacenamiento de agua residual (Fig. 4) para el funcionamiento continuo en la PTAR [15]. Según los parámetros de diseño, con un volumen útil de $224 \mathrm{~m}^{3}$, el tiempo de retención máximo en esta última unidad fue establecido en $0.67 \mathrm{~h}$ [15], pero actualmente este periodo ha llegado a ascender a más de $24 \mathrm{~h}$ entre semana e incluso superar las $96 \mathrm{~h}$ de estancamiento durante los fines y principios de ésta (por falta de producción, fallas mecánicas, retrasos o reprogramación de fábrica), por lo que, fácilmente, los tiempos necesarios para la fermentación parcial de sustratos son inducidos, llegando a una baja del pH en torno a $5.34 \pm 0.23$. La disminución de $\mathrm{pH}$ en el agua residual a medida que pasa por las unidades de pretratamiento y tratamiento primario, ya habían sido detectadas en este sistema de depuración con anterioridad [8].

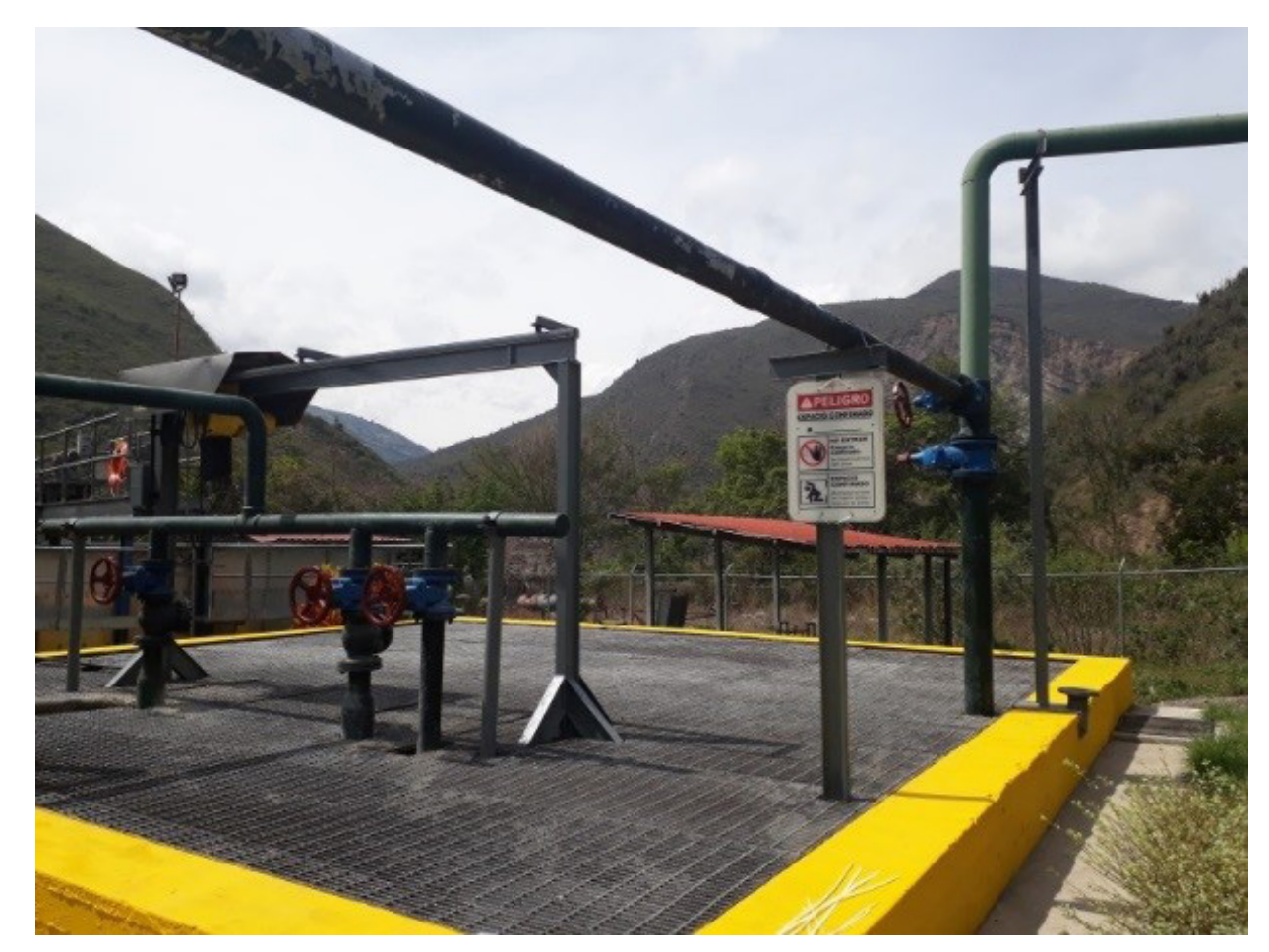

Fig. 4. Vista superior del tanque de igualación u homogeneización. Fuente: autores.

Disminuciones de este parámetro en estanques para el agua residual industrial donde no hay procesos de mezclado o aireación, han sido asociadas a procesos de oxidación anaeróbicas en elevados tiempos de retención [20] y a la capacidad tampón de los efluentes en casos con menor almacenamiento [21]. Otros sistemas industriales registran valores de $\mathrm{pH}$ casi invariables entre unidades de tratamiento [22], incluso teniendo estructuras donde el agua es almacenada durante algún tiempo antes del reactor biológico, lo cual se asocia a bajos tiempos de retención que no generan las condiciones encontradas en el presente estudio. Reducciones mínimas de $\mathrm{pH}$ han sido detectadas en tanques de homogeneización con tiempos de retención promedio de $16 \mathrm{~h}$ para efluentes líquidos industriales [23]. El descenso en el pH puede afectar negativamente los procesos de coagulación química (se discutirá más adelante) y el tratamiento biológico [18].

Otro de los aspectos destacados tiene que ver con el balance de nutrientes usualmente percibido en el tanque de igualamiento u homogeneizador, por cuanto, al hacer una revisión, estos no fueron tomados dentro de las consideraciones del diseño original en [15]. Es bien sabido que las relaciones mínimas de nitrógeno y fósforo que han de existir para satisfacer un tratamiento biológico aerobio deben rondar las cinco y una parte, respectivamente, por cada 100 partes de carbono; es decir, 100/5/1 [14]. De acuerdo a esto, el contenido nutricional dio a conocer que las concentraciones que suelen presentarse en el tanque de igualamiento rondan los $3800 \pm 381 \mathrm{mg} \mathrm{DBO}_{5.20} / \mathrm{L} ; 6.5 \pm 1.5 \mathrm{mg} \mathrm{N} / \mathrm{L}$ y $33 \pm 9 \mathrm{mg} \mathrm{P} / \mathrm{L}$, lo que significa una relación de DBO5.20/N/P de 100/0.17/0.87. Se puede notar, sobretodo, la carencia de la disponibilidad de nitrógeno orgánico, amoniacal libre y salino necesario para satisfacer los requerimientos metabólicos bacterianos del sistema secundario posterior. 
Al comparar con la DBO5.20 de diseño, es decir, $4334 \mathrm{mg} \mathrm{O} / \mathrm{L}$ (Tabla 1), se deduce que aproximadamente $216 \mathrm{mg} \mathrm{N} / \mathrm{L}$ y $43.34 \mathrm{mg} \mathrm{P} / \mathrm{L}$ serían, en promedio, siempre necesarios para respetar las relaciones nutricionales $\mathrm{C} / \mathrm{N}$ y $\mathrm{C} / \mathrm{P}$ mínimas. De esta manera se puede notar que la calidad del agua residual que realmente es tratada en el sistema, es diferente a lo previsto en el diseño.

$\mathrm{Al}$ respecto, se reportaron algunos históricos de los parámetros del agua residual objeto de estudio en esta investigación [8]. Con esos datos se puede calcular en tres muestreos diferentes en el afluente a la PTAR en los años 2017 y 2018, relaciones de concentración de nutrientes DBO5.20/N/P de 100/0.33/0.12; 100/1/0.11 y 100/1.87/0.72, lo cual ratifica que existe un déficit de nitrógeno y fósforo para el tratamiento biológico posterior. El mismo autor encontró concentraciones de nitrógeno promedio de $29.08 \pm 19.58 \mathrm{mg} / \mathrm{L}$, resultando ser, en el mejor de los casos, un $79 \%$ de los $62 \mathrm{mg} / \mathrm{L}$ previstos en el diseño original (Tabla 1) y puede llegar a ser, apenas, un $15 \%$. En el caso del fósforo, se documentó una concentración promedio de $6.75 \pm 2.88 \mathrm{mg} / \mathrm{L}$ en el afluente del sistema de tratamiento [8], siendo mayor a la utilizada en el proyecto de diseño.

Otras empresas con procesos productivos muy similares han reportado concentraciones diferentes. Por ejemplo, en un muestreo puntual se mostró $9.5 \mathrm{mg} / \mathrm{L}$ de nitrógeno total (sustracción entre el nitrógeno Kjeldahl y amoniacal) y $15.6 \mathrm{mg} / \mathrm{L}$ de fósforo [24]. Por supuesto, el caudal de agua residual puede influir en los valores expresados en concentración. De igual manera, la cantidad de materia prima procesada, su calidad e insumos agregados, también pueden afectar la calidad de las aguas residuales, a pesar de ser procesos productivos semejantes.

\section{Sistema de Coagulación, Floculación y Flotación por Aire Disuelto}

Cuenta con una cuba para la mezcla rápida (coagulación), otra para la mezcla lenta (floculación) y un tanque donde se inyecta aire presurizado y ocurre la flotación (Fig. 5). Su objetivo es remover parte de la carga orgánica proveniente de los aceites, grasas libres y emulsionadas, así como los sólidos suspendidos y DBO [15]. El principio de funcionamiento de la unidad depende de la inyección de aire a través de una turbina mecánica y del tiempo de contacto resultante entre el caudal de agua residual y el área superficial dispuesta. Por tanto, al evaluar las condiciones del diseño original; es decir, para caudal igual a $12.16 \mathrm{~L} / \mathrm{s}$ y área superficial de $6.5 \mathrm{~m}^{2}$, la carga hidráulica resultante es de unos $162 \mathrm{~m}^{3} / \mathrm{m}^{2} \cdot \mathrm{d}$; un valor aceptable que se encuentra dentro de las especificaciones para unidades DAF entre 120 y $360 \mathrm{~m}^{3} / \mathrm{m}^{2} \cdot \mathrm{d}$ [14].

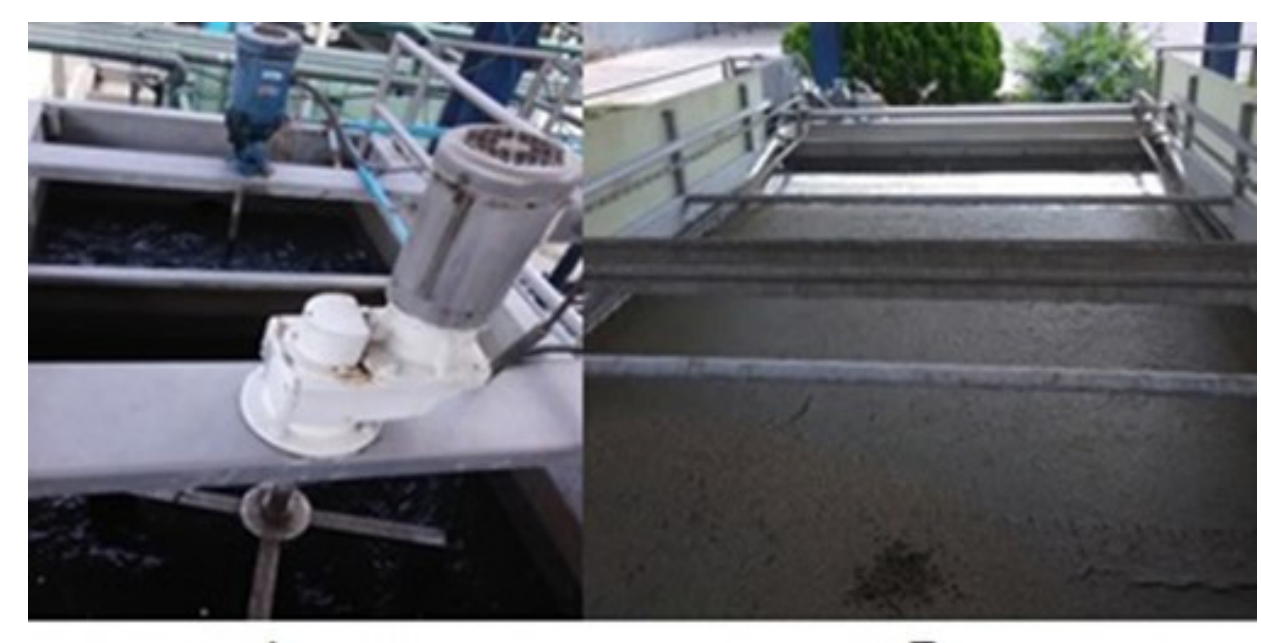

A

B

Fig. 5. Vista superior del tanque de mezcla rápida y lenta (A) y del tanque de flotación (B). Fuente: Autores.

Las mediciones realizadas en la unidad demostraron que el área superficial existente para la flotación coincide con la mencionada por el fabricante y que, de hecho, se induce un efecto positivo en el tratamiento bajo las condiciones actuales de producción en fábrica. Esto debido a que para la media de caudal actual de $1.58 \mathrm{~L} / \mathrm{s}$, la carga superficial desciende a $21 \mathrm{~m}^{3} / \mathrm{m}^{2} \cdot \mathrm{d}$, provocando menor cantidad de agua por unidad de área y un incremento del tiempo de contacto, los cuales conllevan a una mayor remoción de materias en el tanque de flotación. Altas eficiencias de remoción han sido encontradas en tratamientos DAF para vertidos industriales que operan al 50\% de su caudal de diseño [23]. 
No obstante, la significativa reducción del caudal afluente ha desencadenado un perjuicio en la coagulación. En un principio, para la tasa estimada de $12.16 \mathrm{~L} / \mathrm{s}$, los tiempos de contacto propiciados dentro de las cubas de mezcla rápida y lenta eran de 2.46 y 3.5 min respectivamente, pero en la actualidad estos periodos se han extendido a 18 y 27 min para cada caso. El inconveniente no radica en el tiempo de floculación, pues éste se encuentra dentro del intervalo de los 20 y $30 \mathrm{~min}$, pero sí en el de coagulación, dado que el agua es sometida a un proceso de mezclado mayor a $100 \mathrm{RPM}$ por más de los 2 o 3 min recomendados [25]. Este hecho fue comprobado en múltiples ocasiones mediante pruebas en campo y ensayos de jarras, como el de la Fig. 6, que simularon las condiciones de tiempo mencionadas. Adicionalmente, algunas ocasiones se visualizó en el efluente del DAF la presencia de sustancias en la superficie (iridiscencia), lo cual se asoció a coagulante remanente.

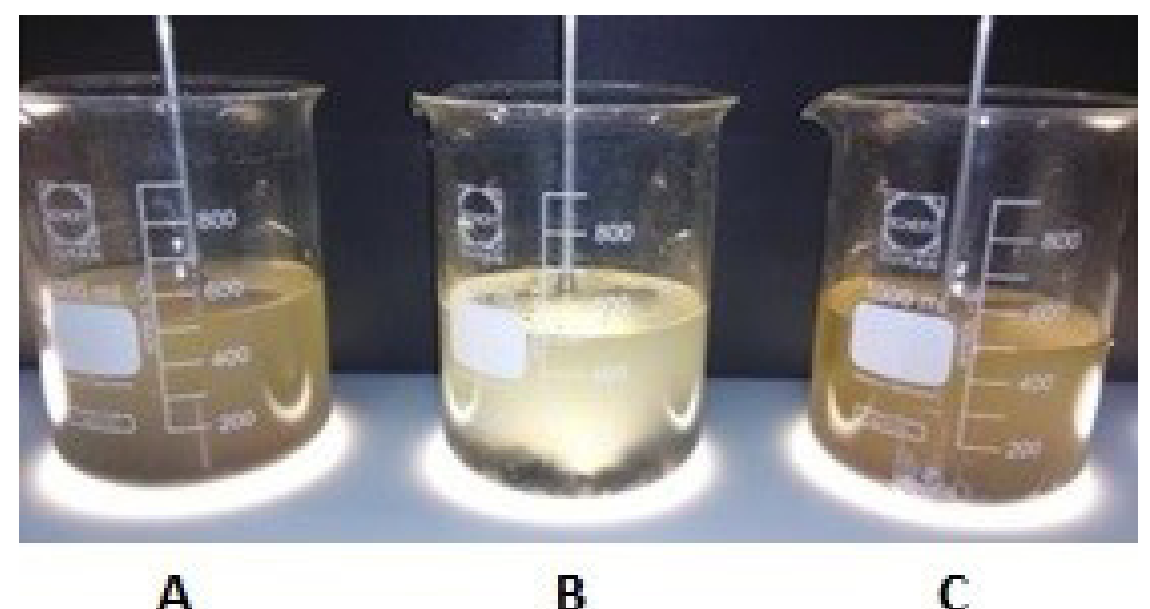

Fig. 6. Rompimiento flocular debido a 150 RPM por 20 min de agitación (A) y conformación flocular por $1 \mathrm{~min}(\mathrm{~B})$, para $3 \mathrm{~mL}$ de $\mathrm{Al}_{2}\left(\mathrm{SO}_{4}\right)_{3}(4 \%)$; sedimentación sin químico $(\mathrm{C})$. Fuente: Autores.

Como se aprecia en la Fig. 6, para la dosis media más usada del coagulante (3 mL de $\mathrm{Al}_{2}\left(\mathrm{SO}_{4}\right)_{3}$ al 4\%) el vaso "B" demuestra una apropiada separación de fases luego de agitación con 150 RPM durante 1 min, mientras que el vaso "A" (150 RPM durante 20 min) siempre demostró rompimiento flocular y un incremento de la turbidez y el color respecto a la misma muestra sometida a sedimentación sin el uso del químico (vaso C). De acuerdo a 30 ensayos realizados, la dosificación de coagulante en la cuba de mezcla rápida representaba un gasto inapropiado por el consumo de energía al aplicarse mezcla por más tiempo del necesario, ocasionando fragmentación de los flóculos, y, sobre todo, por inyectar un químico que lograba altas remociones de materia orgánica y nutrientes, lo cual, a su vez, generaba un desequilibrio al tratamiento biológico.

Ya en el análisis hecho en el tanque de igualación se notó que el pH del agua residual iba disminuyendo como consecuencia de la oxidación anaeróbica parcial que ocurre por los altos tiempos de retención en espacios donde no se tiene aireación. Esta acidificación trae dificultades desde el punto de vista operacional de la PTAR, debido, principalmente, a la incompatibilidad que estas aguas demuestran frente al sulfato de aluminio usado, el cual es un coagulante aplicado ocasionalmente para regularizar las cargas orgánicas hacia el tratamiento biológico y cuyos rangos de efectividad no coinciden con las fluctuaciones existentes de $\mathrm{pH}$.

El pH óptimo para la mejor dosificación de sulfato de aluminio como coagulante está alrededor de 7 (menor cantidad de químico aplicado y valor más bajo de turbidez remanente en el agua) [26]. Sin embargo, de manera genérica se considera un uso adecuado de este químico para aguas cuyos valores de $\mathrm{pH}$ se encuentren entre 4 y 7 [26] o de 5.8 a 7.4 [25]. Fuera de este último rango se encontraron todas las muestras reportadas en la Tabla 2 para el efluente del tanque de igualación (afluente del DAF). Si el pH no es el adecuado, la remoción de turbidez es pobre y se pueden solubilizar los metales presentes en los coagulantes [26]. Otra desventaja es que a bajo $\mathrm{pH}$ en el agua residual, como en el caso del afluente a la cuba de mezcla rápida en el sistema de depuración en estudio, se debe aplicar mayor cantidad de químico para lograr una elevada remoción de turbidez [27]. 
En el caso del pH en la salida del DAF, las mediciones realizadas notaron que la variación con respecto a su afluente era mínima, por lo que no representó un punto que ameritara mayor dedicación. Otras investigaciones detectaron, en promedio, un pequeña disminución de 0.08 entre el afluente y efluente de esta unidad de tratamiento (de 5.86 a 5.78) [8], lo cual se puede asociar al coagulante aplicado.

Por otra parte, en términos de la eficiencia esperada en el DAF, se tuvo que el diseño original manejó proyecciones de remoción de materia orgánica en torno al 40\% como DBO5.20 y del $70 \%$ como sólidos suspendidos totales [15], los cuales son valores similares a los reportados en otros sistemas operativos de este tipo para efluentes industriales (70.66\% para SST y 54.88\% para DBO5.20) [23]. Entonces, según la Tabla 1, teóricamente, las concentraciones pasarían de 8657 a $2597 \mathrm{mg}$ SST/L y de 4334 a $2600 \mathrm{mg}$ DBO5.20/L. Si bien las numerosas pruebas demuestran que el afluente a la unidad realmente exhibe medias de $3800 \pm 381 \mathrm{mg} \mathrm{DBO} / \mathrm{L}$, en éste los SST nunca han sobrepasado los $2500 \mathrm{mg}$ SST/L, de manera que el sistema, por sí solo, siempre ha propiciado una mayor separación de materias que las especificadas en el diseño y con una eficiencia más alta. Esto último debido a que, en el peor de los casos; es decir, para un flujo de $2500 \mathrm{mg} \mathrm{SST} / \mathrm{L}$, generalmente se obtiene un efluente con tan solo $400 \pm 100$ mg SST/L, totalizando un porcentaje máximo del 88 por 100 de eficiencia y del 70 por 100 en aquellos escenarios donde el afluente caracteriza menos de $800 \mathrm{mg}$ SST/L y el efluente exhibe $250 \pm 80 \mathrm{mg}$ SST/L. De hecho, en otros periodos en el mismo sistema objeto de estudio, se han conseguido, en promedio durante varias semanas, llevar de 993.81 a 191,76 mg SST/L, lo cual significa una remoción del $80.62 \%$ [8] que se encuentra dentro del rango observado.

Las tasas de remoción que se midieron en el DAF son similares a las reportadas en otras investigaciones para aguas residuales industriales, como el caso ya mencionado [23] que también tuvo condiciones de trabajo por debajo de su capacidad de diseño. Otro equipo de flotación operativo en campo disminuyó un $86.2 \%$ de la concentración de SST [28]. Celdas de flotación por aire disuelto para vertidos industriales en condiciones de laboratorio, con procesos de coagulación y floculación previos, lograron remover $87 \%$ de concentración para DQO y $85 \%$ de SST [29]; y entre 50.7 y $85.7 \%$ de SST aplicando dos tipos de coagulantes químicos previo a la flotación [30].

Asimismo, los análisis de la relación DQO/SST realizados a las muestras tomadas en el efluente del DAF, arrojaron que por cada miligramo de SST se induce una demanda de $10.9 \pm 1 \mathrm{mg}$ de DQO, lo cual comprueba que se exceden ampliamente los niveles máximos establecidos en el diseño para la salida de la unidad, puesto que para la concentración teórica original de $2597 \mathrm{mg} \mathrm{SST} / \mathrm{L}$, se tendría una DQO por el orden de los $28000 \mathrm{mg} / \mathrm{L}$, excediendo la carga orgánica proyectada para el tratamiento biológico (4000 mg DQO/L).

Esto revela una falla grave en el sistema, pues indica que las concepciones originales para la descarga del DAF que, a su vez, son el afluente del tratamiento secundario, presentan un error de diseño, demostrándose dos aspectos esenciales: (a) las estimaciones iniciales de carga de sólidos y materia orgánica que alimentarían al DAF estuvieron muy alejadas de la realidad de la planta y (b) que, si bien los SST en el agua residual constituyen solo una tercera parte de la concentración proyectada $(8657 \mathrm{mg} / \mathrm{L})$, bajo las condiciones de producción experimentadas desde el año 2016, la separación de materias se da con una eficiente tasa de remoción.

Los porcentajes de eficiencia en el diseño del DAF fueron establecidos, únicamente, sobre el principio del área de flotación, ocurriendo, por tanto, un aumento sustancial de estos últimos durante la inyección de químicos coagulantes. Ciertamente, esta ha sido una de las razones por las cuales, desde mediados del 2018, la dosificación de químico coagulante y floculante fue interrumpida casi en su totalidad; acción que repercutió positivamente tanto en la operación de la planta, como en los costos asociados a su explotación. Hasta antes del periodo mencionado, la adición de policloruro de aluminio y floculante que reducía los niveles de SST hasta $100 \mathrm{mg} / \mathrm{L}$, era una de las exigencias establecidas por la empresa encargada de la operación y mantenimiento de la PTAR para el buen funcionamiento de los reactores. Sin embargo, como la carga orgánica asociada a la aplicación de esta medida operacional reducía los niveles de DQO y DBO a $820 \pm 190 \mathrm{mg} \mathrm{O} / \mathrm{L}$ y $760 \pm 114 \mathrm{mg} \mathrm{O} / 2$ Lespectivamente, se inducían desequilibrios biológicos que magnificaban e incrementaban los niveles de turbidez en el efluente final de la PTAR. 
Asimismo, otro de los inconvenientes descubiertos tuvo que ver con los niveles de nitrógeno y fósforo removidos durante el proceso de coagulación y floculación química, previa al DAF. Como se discutió durante el balance de nutrientes en el tanque de igualación, las relaciones $\mathrm{C} / \mathrm{N}$ en este punto no son las suficientes y las de $\mathrm{C} / \mathrm{P}$ casi son las necesarias para atender las demandas del tratamiento biológico; por lo que la implementación de los químicos, lejos de contribuir con el buen desarrollo del proceso depurativo, lo afectan negativamente por reducir la disponibilidad de los nutrimentos a valores casi nulos. Esto es debido a que para lograr la carga orgánica de trabajo, la captura u adsorción del N y P contenido en el seno de la solución (junto con las materias y natas del área de flotación) también se efectúan y remueven, conllevando a que, hasta la fecha, el efluente del DAF que discurre al reactor de lodos activados exhiba concentraciones de $2.5 \pm 1 \mathrm{mg} \mathrm{N} / \mathrm{L}$ y $9 \pm 4 \mathrm{mg} \mathrm{P} / \mathrm{L}$.

Las concentraciones obtenidas en la salida del DAF son tan bajas que, en el caso del nitrógeno, se cumple la norma de descargas de efluentes líquidos venezolana [31] y, algunas veces, también para el fósforo, aún sin pasar por el tratamiento secundario de la PTAR. Estos valores de nutrientes son similares a los obtenidos en otras aguas residuales industriales tratadas en sistemas completos; es decir, luego de los tratamientos preliminares, primarios, secundarios y, en ciertos casos, terciarios [28], [32], [33].

Aun cuando existe un descenso considerable de los SST y de la DBO en el efluente del DAF (hasta $110 \pm 40 \mathrm{mg} \mathrm{SST} / \mathrm{L}$ y $1360 \pm 420 \mathrm{mg} \mathrm{O} / \mathrm{L}$, respectivamente), las insuficiencias de nutrientes son y siempre han sido una constante en el tiempo, tomando en cuenta estos resultados y los conseguidos [8], que antes fueron mencionados; esto como consecuencia de las malas consideraciones iniciales efectuadas en cuanto a la caracterización de las aguas residuales que se iban a depurar en esta PTAR y a la merma en el caudal y cambios de las características del agua residual en los últimos años. De hecho, la relación $\mathrm{DBO}_{5.20} / \mathrm{N} / \mathrm{P}$ que se puede calcular con las mencionadas concentraciones en la descarga del DAF, arroja 100/0.18/0.66, con lo cual se ratifica que los requerimientos de nutrientes para el posterior tratamiento biológico no están siendo satisfechos.

Con respecto a la mencionada disminución que se logra de DBO5.20 en el DAF, cuantificada en promedio en un 64\% en el presente estudio, para otro estudio [8] se encontró una remoción promedio muy similar de $62 \%$. En el caso de otros DAF para aguas residuales industriales, tambien se reportan eficiencias de 67\% [33] y 54.88\% [23] para la materia orgánica biodegradable. Todo esto permite afirmar que el comportamiento de la unidad DAF para la remoción de la DBO5.20 es eficiente y se encuentra dentro de valores comunes para este tipo de tecnología en el tratamiento de efluentes industriales.

Desde el punto de vista operativo, (a) debido a las bajas concentraciones actuales de los parámetros químicos en el agua residual, si se compara con la calidad esperada en el diseño original; (b) para contar con una mejor calidad al afluente del reactor de lodos activados; y (c) disminuir los gastos asociados al consumo de químicos; se recomendó, como principal medida de mejora, mantener la flotación por aire disuelto pero inactivar los procesos de coagulación y floculación; es decir, sin inyección ni mezcla de sustancias químicas.

No obstante, si debido a cambios en los procesos y cantidad productiva de la industria, el afluente a la PTAR vuelve a alcanzar un caudal y características similares a las contempladas en el proyecto de diseño del sistema, sería necesario efectuar algunas modificaciones en los procesos de coagulación, floculación y flotación. Para dicho escenario, a continuación se especifican las tres alternativas de mejora presentadas a la gerencia de la empresa:

a) Modificación del sistema de dosificación de químico. Esto con la finalidad de inyectar el coagulante directamente en la cuba de mezcla lenta, para lo cual habría que desviar la tubería que transporta el coagulante desde el tanque de mezcla rápida hacia el tanque de mezcla lenta. En algunas contingencias presentadas en el sistema, esto ha demostrado buenos resultados por cuanto no se produce rompimiento flocular.

b) Reducción de los RPM del motor de mezcla rápida. Siendo un hecho comprobado que bajo condiciones de mezcla lenta el tiempo de residencia no afecta negativamente la conformación flocular, reducir las RPM de 150 a 40 permitirá la continuidad de los flóculos formados hasta su remoción fuera de la unidad de DAF. La comprobación de esta alternativa fue efectuada mediante ensayo de jarras, en donde se simuló este escenario sometiendo la muestra a 40 RPM durante más de 30 min. 
c) Desactivación de la mezcla rápida en la unidad. El propósito es detener completamente la mezcla rápida y mantener la agitación lenta únicamente como proceso funcional; implicando la inyección de químico en la cuba de mezcla rápida (sin agitación) para que, mediante rebose, pase directamente hacia el área de mezcla lenta y la coagulación-floculación ocurra progresivamente.

Es cierto que las anteriores alternativas parecen cuestionar lo que teóricamente se recomienda para los procesos de coagulación y floculación, específicamente de que sean procesos por separado, con sus respectivos mezclados y puntos de dosificación [25], [26]. Sin embargo, cuentan con verificación probada en campo en el sistema de depuración, lo cual puede deberse a la afinidad de esta agua industrial particular a los químicos dosificados o al mezclado inducido por el equipo y configuración de paletas existente en la cuba de mezcla lenta; son aspectos inusitados que ocurren en el funcionamiento de las PTAR y que requieren una investigación más detallada. Adicionalmente, ya se hizo mención que son opciones a implementar en dado caso que aumente la producción en fábrica y se tengan mayores caudales de agua residual a tratar. La mejor opción para las actuales condiciones existentes consiste en desincorporar ambos procesos del funcionamiento de la PTAR, tal como ya se indicó.

En el caso de mantener las condiciones que favorecen la disminución del $\mathrm{pH}$ y la permanencia de la coagulación y floculación, podría analizarse la opción de usar reactivos químicos cuyo desempeño sea más favorable en medios ácidos, como el cloruro férrico dentro de la línea de los coagulantes convencionales [25]. Otra opción que anteriormente ha mostrado buenos resultados en la PTAR, es el uso de coagulantes inorgánicos prepolimerizados como los policloruros de aluminio, el cual, en su momento, demostró mejor adaptación a las fluctuaciones de pH del agua residual, se utiliza menor cantidad de químico y logra mejor eficiencia de remoción [34]. Todo lo anterior se ha reflejado en investigaciones realizadas que comparan el uso del sulfato de aluminio versus los policloruros de aliminio [35].

Otra acción operativa implementada y probada últimamente en la PTAR, es el funcionamiento por cargas o lotes (batch) del sistema de coagulación, floculación y flotación. Al respecto, en ciertos estudios se indica que son reactores donde el afluente y efluente son discontinuos [14]; se permite el ingreso del agua residual, al alcanzar el volumen requerido se detiene el llenado, se activa el proceso de tratamiento deseado, se transcurre el tiempo previsto para las reacciones depurativas y, terminada dicha duración, se vacía el agua residual tratada. Suelen usarse poco en la depuración en campo, siendo más comunes para sistemas pequeños o de poca capacidad [14], debido al intermitencia de la descarga de aguas residuales y bajos caudales. Esto último es, precisamente, lo que se aprovechó en la PTAR en estudio, por cuanto son más reiterativos los lapsos donde no hay producción en la fábrica y, por ende, no hay agua residual. El tratamiento y la eficiencia del sistema DAF con inyección de químicos, no se ha visto afectado negativamente por esta acción operativa. De no cambiar las condiciones actuales de fabricación, esta forma de funcionamiento pudiera adaptarse y tomarse como permanente.

Corresponde a la empresa tomar la opción que mejor consideren en función de las experiencias probadas en esta investigación.

Esta investigación logra mostrar cómo cotejar el diseño de un sistema de tratamiento con la realidad operativa del mismo, así como el hecho de manifestar los inconvenientes y posibles alternativas de mejora cuando se tiene un agua residual afluente que se aleja, por diversas razones, del caudal y características previstas o estimadas inicialmente en el proyecto original. Adicionalmente, desde el punto de vista teórico, sustenta la importancia de concebir apropiadamente las características del agua residual al momento del diseño y, también, deja abierta la posibilidad de que bajo ciertos escenarios, las aguas residuales pueden depurarse en un sistema de coagulación, floculación y flotación por aire disuelto realizando cambios en la forma y puntos de dosificación y mezclado, lo cual requiere mayor profundidad de experimentación y análisis.

\section{CONCLUSIONES}

Se realizó la evaluación del tratamiento preliminar y primario de la planta de tratamiento de agua residual industrial en estudio, encontrándose que la merma en los procesos productivos para elaborar los alimentos ha repercutido en el caudal y características del efluente líquido 
residual. Estas variaciones han provocado aumento de los tiempos de retención en el tanque de bombeo y en el tanque de igualación ( $\mathrm{u}$ homogeneizador), generando disminuciones de $\mathrm{pH}$ en el agua residual, las cuales se derivan de procesos de descomposición de la materia orgánica. Asimismo, el sistema de coagulación, floculación y flotación por aire disuelto logra remover elevados porcentajes de materia orgánica y nutrientes, hasta tal punto que ocasionan un perjuicio al tratamiento biológico posterior.

Según los resultados obtenidos de la dinámica real del agua residual, se comprueba que las concepciones establecidas en el proyecto de diseño original, específicamente las características teóricas asumidas para el efluente industrial, fueron erradas y se alejan de lo que realmente ocurre.

Por lo anterior, es necesario implementar medidas de mejora para inactivar o modificar la coagulación y floculación en la PTAR, las cuales generarán reducción de los costos de operación y mantenimiento y un afluente adecuado para la dinámica microbiológica del tratamiento secundario

\section{Financiamiento}

Artículo de investigación científica derivado del proyecto de "evaluación del diseño de la planta de tratamiento de aguas residuales y propuesta de reacondicionamiento del sistema de lodos activados de PepsiCo, planta La Grita, estado Táchira”, el cual fue financiado por PepsiCo Alimentos S.C.A. Dicho proyecto se desarrolló entre julio 2018 y marzo 2019.

\section{Agradecimientos}

Se agradece a la empresa ALFATLANTIC C.A. como contratista encargada de la operación y mantenimiento de la PTAR en estudio, así como a la empresa multinacional propiedad de las instalacionales

\section{REFERENCIAS}

[1] A. Noyola, J. M. Morgan-Sagastume \& L. P. Güereca, Selección de Tecnologías para el Tratamiento de Aguas Residuales. CDMX: UNAM, 2013. Available: http://www.pronatura-sur.org/web/docs/Tecnologia_Aguas_Residuales.pdf

[2] C. Suher, J. Almarza, F. Pedrique, C. Cárdenas \& L. Herrera, "Optimización del proceso de tratamiento de aguas residuales de una industria cervecera," Interciencia, vol. 34, no. 11, pp. 764-770, Nov. 2009. Available: https://www.interciencia.net/wp-content/uploads/2018/01/764-YABROUDI-7.pdf

[3] B. Henry, J. Monge, J. Moscoso, S. Oakley, L. Salguero y P. Saravia. Tratamiento de Aguas Residuales Domésticas en Centroamérica. Un Manual de Experiencias, Diseño, Operación y Sostenibilidad, 2 ed, Gt, USA: USAC, 2011. Available: http://www.da.go.cr/wp-content/uploads/2017/01/Manual-Tratamiento-Aguas-Residuales-en-CA.Final_.06.06.11.pdf

[4] M. M. Ronces, "Evaluación de funcionamiento de plantas de tratamiento de aguas residuales de un mucipio del sureste de México," Proyecto grado, dpto Quím, UAEMX, Toluca, Mx, 2018. Available: http:// hdl.handle.net/20.500.11799/94946

[5] Y. Pineda \& A. Avalos, "Evaluación de la Planta de Tratamiento de Aguas Residuales del municipio de La Palma, departamento de Chalatenango, El Salvador," Proyecto grado, dpto Cs Agr, UES, SV, 2019. Disponible en http://ri.ues.edu.sv/id/eprint/19737

[6] G. Ramírez \& J. Tutillo, "Evaluación hidráulico - sanitaria de la planta de tratamiento de aguas residuales de la comunidad Buena Esperanza, parroquia Cangahua, cantón Cayambe, provincia Pichincha," Proyecto grado, dpto Cs Fis Mat, UCE, UIO, 2018. Disponible en http://www.dspace.uce.edu.ec/ handle/25000/16226

[7] M. Y. de la Vega, Eficiencia en plantas de tratamiento de aguas residuales. Contribución a la gestión $y$ desarrollo social. MX DF, MX: Refugia, 2012. Disponible en http://indesol.gob.mx/cedoc/pdf/III.\%20 Desarrollo\%20Social/Agua\%20y\%20Saneamiento/Eficiencia\%20en\%20Plantas\%20de\%20Tratamiento\%20de\%20Aguas\%20Residuales.pdf

[8] C. Mora, "Propuesta de mejora para una planta de tratamiento de aguas residuales industriales de una empresa de producción de alimentos ubicada en La Grita, municipio Jáuregui, estado Táchira," Proyecto grado, dpto Ing Amb, UNET, Táchira, VE, 2019. Disponible en http://curly.unet.edu.ve/sicpro/ publico/proyectos?sort=FechaFin.desc\&page $=15$

[9] APHA, AWWA \& WPCF, Métodos normalizados para el análisis de aguas potables y residuales. 17 Ed. MAD, ES: Ediciones Díaz de Santos, 1992.

[10] WTW, “Oxitop(r) respirometeres,” [online], Xylem Inc, Rye Brook, NY, USA, 2019. Available: https:// www.xylemanalytics.com/en/products/oxitop-respirometers

[11] Hach, "Colorímetros," [online], Hach Co, LVLD, USA, 2021. Available: https://es.hach.com/quick. search-quick.search.jsa?keywords=Color\%C3\%ADmetros 
[12] A. Pulido, L. Sánchez \& M. Cárdenas, “Tratamiento de agua por coagulación floculación,” Práctica No. 03, Táchira, VE: UNET, Mar. 2017.

[13] Metcalf \& Eddy, Ingeniería de Aguas Residuales. Tratamiento, vertido y reutilización. 3 Ed, vol. 1-2, MAD, ES: McGrall-Hill, 1995.

[14] J. A. Romero, Tratamiento de Aguas Residuales. Teoría y principios de diseño. Bog, Co: Escuela Ing, 1999.

[15] Diseños Ambientales C.A., Ampliación de la planta de tratamiento de aguas residuales industriales y domésticas de Snacks América Latina, La Grita, estado Táchira, Tachira, VE: DISA, 2006.

[16] P. Poirrier, "Hidrólisis y acidificación psicrófila de moléculas complejas en sistemas anaerobios," Tesis doctoral, dpto Ing Quím, USC, SCQ, ES, Dic. 2005. Recuperado de https://www.usc.es/biogroup/sites/ default/files/PaolaPoirrier.pdf

[17] Y. Lorenzo y M. Obaya, "La digestión anaerobia. Aspectos teóricos. Parte I," ICIDCA, vol. 39, no. 1, pp. 35-48, Ene. 2005. Recuperado de https://www.redalyc.org/pdf/2231/223120659006.pdf

[18] R. Parra, "Digestión anaeróbica: mecanismos biotecnológicos en el tratamiento de aguas residuales y su aplicación en la industria alimentaria," Producción + Limpia, vol. 10, no. 2, pp. 142-159, Jul. 2015. Disponble en http://repository.lasallista.edu.co/dspace//handle/10567/1436

[19] M. Ramos, G. Rodríguez, R. Santiago, S. Alemán, O. Castillo \& A. Perales, "Production and characterization of a snack based on maize flour and Atlantic mackerel (Scomber scombrus)," CyTA - Journal of Food, vol. 17, no. 1, pp. 1006-1013, Dic. 2019. https://doi.org/10.1080/19476337.2019.1690584

[20] C. España, "Análisis de eficiencia y funcionamiento de la planta de tratamiento de aguas residuales de la empresa Lácteos Andinos de Nariño LDTA," Proyecto grado, UIS, SDR, CO, 2008.

[21] K. Pimiento, "Evaluación de la planta de tratamiento de aguas residuales de una empresa dedicada a la elaboración de productos de origen lácteo," Proyecto grado, dpto. Ing Amb, UNET, Táchira, VE, 2017.

[22] Y. Caldera, E. Gutiérrez, M. Luengo, J. Chávez \& L. Ruesga, "Evaluación del sistema de tratamiento de aguas residuales de industria avícola," Rev Cient, vol. XX, no. 4, pp. 409-416, Jul-Agos. 2010. Disponible en https://produccioncientificaluz.org/index.php/cientifica/article/view/15581

[23] O. I. Gallegos, "Evaluación del sistema de tratamiento de aguas residuales de una planta de procesos avícola," Proyecto grado, dpto Ing Quím, UNI, Mga, NI, 2012. Recuperado de http://ribuni.uni.edu. ni/623/1/37985.pdf

[24] K. A. Ayala, "Evaluación de procesos para la remoción de iones de cloruro en el agua residual de la planta de alimentos PepsiCo (Funza)," Proyecto grado, dpto Ing, Uniamerica, Bog, Co, 2016. Disponible en http://52.0.229.99/handle/20.500.11839/580

[25] J. Suárez, A. Jácome \& P. Ures, Coagulación-Floculación, UDC, ES, FT-PRI-001, Jul. 2014. Available: $\quad$ https://www.wateractionplan.com/documents/177327/558161/Coagulaci\%C3\%B3nfloculaci\%C3\%B3n.pdf/b59be3a9-558c-62c3-66e1-d89f82e3aae7

[26] J. A. Romero, Calidad del Agua. Mx, D.F. MX: Escuela Colombiana de Ingeniería, pp. 67-71, 2002.

[27] C. M. Luna, "Influencia del pH en la coagulación-sedimentación primaria de aguas residuales de un camal," Proyecto grado, dpto Ing, UNAC. Cll., Cll., Pe, 2017. Disponible en http://repositorio.unac.edu. pe/handle/UNAC/2244

[28] M. E. Landi, "Evaluación técnica de la planta de tratamiento de aguas residuales de una industria láctea," Proyecto grado, Esc. Form. Tecn, EPN, UIO, EC, 2018. Disponible en http://bibdigital.epn.edu. ec/handle/15000/19553

[29] A. M. Bolaño, "Sistema de flotación por aire disuelto para el tratamiento de aguas residuales industriales," Proyecto de grado, EIA, ENV, CO, 2009. Recuperado de https://repository.eia.edu.co/bitstream/11190/1918/1/Bola\%C3\%B1oAlberto_2009_SistemaFlotacionAire.pdf

[30] Y. Caldera, M. Sánchez \& E. Gutiérrez, "Calidad física de aguas residuales de una industria avícola en un sistema de flotación por aire disuelto con coagulantes," Rev Tecn URU, no. 13, pp. 57-66, Jul.-Dic. 2017. Disponible en http://uruojs.insiemp.com/ojs/index.php/tc/article/view/429

[31] República Bolivariana de Venezuela, "Normas para la Clasificación y el Control de la Calidad de los Cuerpos de Agua y Vertidos o Efluentes Líquidos," Decreto 883, Gaceta Oficial No. 5021 Extraordinario, Dic. 18, 1995

[32] M. J. del Campo y E. Castelló, "Análisis de la operación de sistemas de remoción de nutrientes en efluentes industriales," presentado en el X Cong. Nac. del Cap. Uruguayo, AIDIS, MVD, URU, pp, 28 29 Ago. 2019. Recuperado de https://aidis.org.uy/img/trabajos-tecnicos-congreso-2019/17-DelCampo. pdf

[33] A. M. Márquez \& E. Guevara, "Descripción y evaluación del funcionamiento de un sistema de tratamiento de aguas residuales en una industria avícola," Rev Ing UC, vol. 11, no. 2, pp. 92-101, Ago. 2004. Recuperado de http://servicio.bc.uc.edu.ve/ingenieria/revista/v11n2/11-2-9.pdf

[34] E. Medina, K. Pimiento, J. Rozo \& O. Rodríguez, "Resultados sobre test's de jarras comparativo de coagulante: Policloruro de Aluminio (PAC) \& Sulfato de Aluminio (SAL) para el tratamiento de aguas residuales industriales de PepsiCo alimentos S.C.A, Planta La Grita," PepsiCo y Alfa Atlantic, Táchira, VE, Informe, Sep. 2018.

[35] J. M. Cogollo, "Clarificación de aguas usando coagulantes polimerizados: caso del hidroxicloruro de aluminio," Dyna, vol. 78, no. 165, pp. 18-27, Feb. 2011. Disponible en https://revistas.unal.edu.co./ index.php/dyna/article/view/25636/39133 
Kleiver Pimiento es Venezolano, Ingeniero Ambiental egresado de la Universidad Nacional Experimental del Táchira (UNET). Cuenta con experiencia técnico-práctica y experimental en el tratamiento de aguas residuales y potables, la gestión de residuos sólidos y el uso de energías alternas en grandes industrias de alimentos y medianas empresas, donde, además, ha desempeñado funciones como investigador, proyectista, auditor y evaluador de campo. Ha sido responsable de proyectos destinados al desarrollo socioambiental y económico de comunidades que ameritan soluciones de índole ingenieril. Se ha desempeñado como conferencista y ponente en diversos eventos académicos nacionales y binacionales en el área de ciencia y tecnología, tanto en la UNET como en otras universidades venezolanas como la Universidad del Zulia (LUZ). https://orcid.org/0000-0001-9780-8425

Marcos José Cárdenas González es Venezolano, Ingeniero Ambiental (UNET), diplomado en Construcción Civil (IUFRONT), escolaridad culminada en la Maestría en Gerencia Ambiental (UNEFA). Ingeniero de Proyectos de Bombas Táchira C.A. (2009-2012). Perteneciente al personal académico adscrito al departamento de Ingeniería Ambiental de la Universidad Nacional Experimental del Táchira. Extensionista e investigador en el tratamiento de aguas residuales domésticas e industriales en el Laboratorio de Investigación Ambiental y Desarrollo Sostenible (UNET). Jefe del Núcleo de Ingeniería del departamento de Ingeniería Ambiental (UNET, 2013-2018). Coordinador de Investigación en Ciencias Exactas y Naturales del Decanato de Investigación (UNET, 2018-2019) y Coordinador de Transferencia y Comercialización de la misma dependencia. Organizador y ponente de eventos académicos locales y nacionales, autor de artículos científicos, tutor y jurado evaluador de pasantías profesionales y proyecto especial de grado; todos principalmente, en el área de tratamiento de agua. https://orcid. org/0000-0001-9780-8425 